\title{
NOVEDADES SOBRE LA DISTRIBUCIÓN DE COLLETIA SPINOSISSIMA, PTEROCAULON BALANSAEY SACCHARUM TRINII EN TANDILIA (BUENOS AIRES) Y SUS POSIBLES CAUSAS
}

\author{
Novelties on the distribution of Colletia spinosissima, Pterocaulon balansae and Saccharum \\ trinii in Tandilia (Buenos Aires) and their possible causes
}

CARLOS O. D'ALFONSO'10, ROSA L. SCARAMUZZINO1@ \& MARCELO L. GANDINI $11^{1,2}$

\begin{abstract}
Resumen: Este trabajo aporta nuevos datos sobre la distribución geográfica de algunas especies nativas del norte argentino: Colletia spinosissima (Rhamnaceae), Pterocaulon balansae (Asteraceae) y Saccharum trinii (Poaceae). A partir del análisis de registros de colectas, bibliográfico y de bases de datos en línea se establece como límite austral de su rango al Sistema orográfico de Tandilia (provincia de Buenos Aires, Argentina), también denominado "Sierras Septentrionales de la Provincia de Buenos Aires". Se plantean posibles causas de la presencia de las poblaciones marginales de las tres especies en el sistema serrano, entre ellas el cambio climático, factores antrópicos y ausencias metodológicas.
\end{abstract}

Palabras clave: Especies nativas, límite austral, norte argentino.

Summary: This report provides new data about the geographical distribution of some native species from nothern Argentina as Colletia spinosissima (Rhamnaceae), Pterocaulon balansae (Asteraceae) and Saccharum trinii (Poaceae). Based on the analysis of collection records, bibliographic records and on-line databases, the orographic System of Tandilia (also called "Sierras Septentrionales de Buenos Aires") is established as the southern limit of their range. We propose some possible causes of the presence of peripheral populations of these three species in this hilly system, such us climate change, anthropogenic factors and methodological absences.

Key words: Native species, Northern Argentina, southern limit.

\section{Introducción}

El sistema orográfico de Tandilia, también denominado "Sierras Septentrionales de la Provincia de Buenos Aires", es uno de los dos cordones serranos de la región pampeana. Se ubica en el centro-sur de la provincia de Buenos Aires $\left(36^{\circ} 30^{\prime}-38^{\circ} 10^{\prime} \mathrm{S}, 57^{\circ} 30^{\prime}\right.$ $61^{\circ} \mathrm{W}$ ), con rumbo NO-SE, abarcando un área de $350 \mathrm{~km}$ por $60 \mathrm{~km}$, con afloramientos de origen Precámbrico y Paleozoico (Cingolani, 2011). En un perfil longitudinal forma un arco abierto cuyas alturas máximas (524 m s.n.m.) se encuentran en las zonas medias del arco y los bloques más bajos en los extremos. Representan un típico sistema de montañas de bloques, separados por fallas y alineados según ellas (Teruggi et Kilmurray, 1980). Dado que no constituyen un cordón continuo, sino que está interrumpido por amplios valles, abras o trechos de llanuras, se han considerado grupos orográficos menores cuyos nombres derivan de las localidades próximas a ellos. Los tres sectores principales del sistema orográfico

\footnotetext{
${ }^{1}$ Facultad de Agronomía, Universidad Nacional del Centro de la Provincia de Buenos Aires. Casilla de Correo 47, 7300 Azul, Buenos Aires, Argentina. E-mail: dalton@faa.unicen.edu.ar

${ }^{2}$ Comisión de Investigaciones Científicas (CIC), 1900 La Plata, Buenos Aires, Argentina.
} 
de Tandilia de NO a SE son: las Sierras de Olavarría, las Sierras de Azul y Tandil y las Sierras de Lobería, Balcarce y Mar del Plata.

Aunque su flora ha llamado la atención a botánicos del siglo XIX (Doering et Lorentz, 1916; Ollerton et al., 2012), cuando aún las transformaciones antrópicas de la matriz circundante eran incipientes, los endemismos son escasos y muchas especies son comunes con otras regiones biogeográficas sudamericanas (Frangi, 1975; Echeverría et al., 2017); probablemente su singularidad se deba a que en el área se encuentran poblaciones periféricas de muchas especies vegetales y en muchos casos poblaciones disyuntas. Entre las aproximadamente 500 especies nativas que habitan Tandilia, varias se distribuyen en el norte del país y se ha reconocido su presencia en estas sierras. En algunos casos junto con el otro sistema serrano bonaerense, Ventania, constituyen el límite austral, por ejemplo para Hypericum connatum Lam. (Cabrera, 1965; Robson, 1990) y Agalinis genistifolia (Cham. \& Schltdl.) D'Arcy (Cabrera, 1965); en otros casos, las poblaciones marginales se hallan en Tandilia y en la llanura interserrana entre Tandilia y Ventania, como Rhodophiala bifida (Herb.) Traub (Cabrera, 1968; Villamil et Martínez, 2014) y, para otras especies, las poblaciones periféricas sólo se encuentran en el Sistema de Tandilia como Criscia stricta (Spreng.) Katinas (Katinas, 1994) o Pamphalea heterophylla Less. (Katinas et Crisci, 2008). En varios casos se ha considerado la distribución geográfica de especies nativas sin incluir su presencia en Tandilia. Puede deberse a que se hallan en sitios poco accesibles para la colección botánica, en su mayoría en propiedades privadas, o de poblaciones muy pequeñas, restringidas a algunos ambientes. De modo que se ha considerado el límite sur de distribución en el norte de la provincia de Buenos Aires, en la ribera del Río de la Plata y en el Delta del Paraná o se ha mencionado a la provincia de Buenos Aires sin señalar un límite preciso de distribución.

Es importante conocer la distribución de las especies nativas en Tandilia para poder comprender tanto el rol de este sistema serrano en la biogeografía de la región pampeana como la presencia de especies subtropicales. Podría hipotetizarse que se trata de expansiones de rango de distribución debidas al cambio climático actual (IPCC, 2018), como se ha considerado el avance hacia el sur de especies vegetales y animales dentro de la provincia de Buenos Aires (Guerrero et Agnolin, 2016; Apodaca et Guerrero, 2019), o a cambios producidos en un pasado relativamente reciente como la formación de la selva marginal de Punta Lara, probablemente en los siglos XVIII y XIX (Guerrero et al., 2018), pero también podrían ser poblaciones relictuales del Pleistoceno Medio (0,780-0,126 Ma). El sistema de Tandilia ha sido considerado un relicto del Espinal (Frenguelli, 1941). Podría ser refugio de paleoflora, que podría asociarse con lo que se ha denominado en otros países (aunque tal vez para mayores alturas y afloramientos más aislados) islas del cielo o "sky islands" (Popp et al., 2008), caracterizadas por la presencia de especies relictuales. Estas pequeñas áreas continentales aisladas, con vegetación diferente a la matriz que las rodea, son denominadas "islas virtuales", en referencia a la teoría de "biogeografía de islas" (MacArthur et Wilson, 1969), pero ninguna de las hipótesis relacionadas con esta teoría han sido aún puestas a prueba para las sierras de Tandilia. Como otros roquedales, por su heterogeneidad microambiental (Speziale et Ezcurra, 2014), podría albergar relictos de una paleoflora antes extendida y actualmente restringida a ambientes más cálidos y septentrionales, como también en el futuro ser refugio para las especies afectadas por cambios en las condiciones climáticas. Por otro lado, junto con el sistema de Ventania y la llanura interserrana constituye el "Positivo bonaerense" (Zárate et Rabassa, 2005; Zárate, 2010), zona que no fue afectada por las transgresiones marinas del Mioceno y posteriores, por lo que puede ser un "refugio seco" al ingreso de las aguas.

En las últimas décadas ha podido coleccionarse en el sistema de Tandilia, en particular en las sierras de Azul, algunas especies no citadas previamente para esta región, entre ellas Colletia spinosissima J. F. Gmel. (Rhamnaceae), Pterocaulon balansae Chodat (Asteraceae) y Saccharum trinii (Hack.) Renvoize (Poaceae). Colletia spinosissima es un arbusto espinoso, subáfilo, con ramas 
sin nudos marcados por una línea transversal, espinas cilíndricas, hojas elípticas u ovadas pequeñas, flores con tubo floral urceolado, con estambres subinclusos y fruto tricoco (Tortosa, 1989, 1995). Pterocaulon balansae es una hierba perenne, de elevada altura, con hojas glabrescentes en el haz y densamente lanosas en el envés; sus capítulos están dispuestos en espigas laxas reunidas en amplias panojas y frutos con papus blanquecino (Cabrera et Ragonese, 1978; Freire et Iharlegui, 2015). Finalmente, $S$. trinii es una hierba perenne, rizomatosa, con hojas lineales de 40 a 100 $\mathrm{cm}$, sumamente escabrosas en los bordes; presenta una panoja densa de $30-40 \mathrm{~cm}$, a veces incluida en una espata de color cobrizo (Molina, 1981); y las espiguillas se disponen de a pares, una sésil y otra pedicelada, ambas cubiertas de una abundante pilosidad que a priori favorecen su dispersión anemócora. Estas especies no son estrictamente rupícolas dado que en otras regiones ocupan otro tipo de ambientes: $P$. balansae se halla en terrenos bajos y húmedos (Freire et Iharlegui, 2015), $S$. trinii en pastizales húmedos y marismas (Welker et al., 2015), y C. spinosissima, si bien ocupa suelos rocosos, cerros y faldeos de montañas, también se ubica en bancos de conchilla y barrancas de río (Tortosa, 1995).

El objetivo de este trabajo es aportar nuevos datos sobre la distribución geográfica de estas tres especies en la región pampeana, en particular en el Sistema serrano de Tandilia, así como plantear algunas posibles causas sobre esas poblaciones marginales y establecer el límite sur de su rango de distribución.

\section{Materiales y Métodos}

Los materiales analizados fueron recolectados en las Sierras de Azul, Sistema de Tandilia, provincia de Buenos Aires, (37 $04^{\prime} \mathrm{S}$, $\left.59^{\circ} 45^{\prime} \mathrm{W}\right)$, durante diversos relevamientos florísticos efectuados entre 1989 y 2019. Los ejemplares se depositaron en el herbario FAA (Thiers, 2019). Fueron determinados con floras y revisiones de géneros (Cabrera, 1965, 1968; Cabrera et Ragonese, 1978; Molina, 1981; Tortosa, 1989, 1995; Freire et Iharlegui, 2015). Adicionalmente para establecer el límite sur de la distribución geográfica de las especies se consultaron las bases de datos Flora Argentina (2019), Portal de Datos Biológicos (2019) y Tropicos (2019) y otros trabajos (Parodi 1940a, 1940b; Cabrera, 1941; Burkart, 1957; Vervoorst, 1967; Alonso et al., 2009; Bayón et Hurrell, 2013; Echeverría et al., 2017).

\section{Resultados}

\section{Colletia spinosissima J. F. Gmel. (Fig. 1A-C)}

Ha sido observada y coleccionada en varios cerros de las Sierras de Azul, con registros no publicados desde 1989 hasta el presente. Forma arbustales que ocupan áreas de diferentes tamaños (Fig. 1A), en algunos casos consociados con Baccharis dracunculifolia DC. ssp. tandilensis (Speg.) Giuliano, B. tridentata Vahl, Berberis ruscifolia Lam., Discaria americana Gillies \& Hook., y la exótica Genista monspessulana (L.) L. A. S. Johnson, entre otros arbustos. Se ubican principalmente próximos a vías de escurrimiento con agua permanente y cauces esporádicos. También existen registros de esta especie para el partido de Azul en SI (Flora Argentina, 2019) y Laguna de los Padres (Pdo. Gral. Pueyrredón) en MO (Trópicos, 2019). Asimismo ha sido mencionada en la Sierra La Barrosa, Pdo. Balcarce (Alonso et al., 2009), y en la Reserva Paitití, Pdo. General Pueyrredón (Echeverría et al., 2017). Su presencia en Tandilia no sería debido a un avance de las últimas décadas dado que en 1883 fue observada por Holmberg (1884) en las cercanías del cerro El Sombrerito (Pdo. Benito Juárez), donde fue coleccionada también con posterioridad en 1966 (Portal Datos Biológicos, 2019).

Habita Ecuador, Perú, Bolivia, noroeste y centro de Argentina: Salta, Jujuy, Tucumán, La Rioja, Catamarca, Córdoba, San Luis, San Juan, Mendoza, Corrientes, Entre Ríos y Buenos Aires (Tortosa, 1989, 1995). En Buenos Aires sólo se ha considerado su extensión hasta la ribera del Río de la Plata, en los partidos de La Plata, Magdalena y Zárate (Tortosa, 1989), donde es un elemento arbustivo característico de los bosques de tala (Cabrera, 1976). Más recientemente también se ha coleccionado en 
el partido de la Costa (Flora Argentina, 2019).

Por lo tanto, el límite sur de esta especie no sería la ribera del Río de la Plata (Tortosa, 1989, 1995) sino el Sistema de Tandilia.

\section{Material examinado: ARGENTINA.}

Buenos Aires: Partido de Azul, Ea. Los Ángeles, Sierras de Azul, 37 08' 08,13'S, 59 44'00,51'W, 29-V-1987, Borzone et al. s.n. (FAA 789); Ea. La Argentina, Sierras de Azul, $37^{\circ} 08^{\prime} 45,75^{\prime} \mathrm{S}, 59^{\circ} 45^{\prime} 10,59^{\prime} \mathrm{W}, 21-\mathrm{IV}-$ 1998, Orfila \& D'Alfonso s.n. (FAA 3324); Ea. La Crespa, Sierras de Azul, 37 04' 55,53'S, $59^{\circ} 4^{\prime}$ '02,56'W, 18-X-2013, D'Alfonso et al. s.n. (FAA 8505); ib. $37^{\circ} 04^{\prime}$ 38,31"S, 59 49' 13,90'W, 23-X-2014, D'Alfonso \& Scaramuzzino s.n. (FAA 8246).

\section{Pterocaulon balansae Chodat (Fig. 1D)}

Habita el sur de Brasil, Paraguay, Uruguay y nordeste de la Argentina (Freire et Iharlegui, 2015), donde crece en tierras bajas y húmedas (Cabrera et al., 2000; Bayón et Hurrell, 2013). Su límite austral de distribución geográfica probable había sido determinado en Punta Lara (Cabrera et Ragonese, 1978), donde se la ubicó en los márgenes del bosque (Cabrera et Dawson, 1944). En la provincia de Buenos Aires se la ha hallado en los partidos del noreste: Zárate, Campana, Tigre, Quilmes, Pilar, Ensenada (Cabrera, 1941; Cabrera et al., 2000), con registros en pajonales, cardasales (predominan especies del género Eryngium), praderas y bosques inundables del Delta y de la ribera platense (Burkart, 1957; Cabrera, 1963; Cabrera et Zardini, 1978).

Ha sido hallada en un arroyo serrano en 1995, aunque no se ha podido acceder nuevamente al sitio para verificar su presencia actual ni ha sido coleccionado en otros cursos de agua de las sierras de Azul. Probablemente se trate de la misma especie citada para el sudeste del sistema, en la Reserva Paitití (Gral. Pueyrredón) como $P$. cordobense Kuntze (Echeverría et al., 2017). Por lo tanto, el Sistema de Tandilia constituiría el límite sur de distribución de esta especie y no Punta Lara (Cabrera et Ragonese, 1978).

Material examinado: ARGENTINA. Buenos Aires: Partido de Azul, Sierras de
Azul, Ea. Santa Rosa, en margen curso de agua, 37 06' 58,91'S, 59 41' 34,10"W, 28II-1995, Orfila \& D'Alfonso s.n. (FAA 2036).

3. Saccharum trinii (Hack.) Renvoize (Fig. 1 E-F)

Coleccionada desde 1994 hasta el presente en las sierras de Azul, en dos cerros que limitan el abra denominada Boca de las Sierras $\left(37^{\circ}\right.$ $\left.03^{\prime} \mathrm{S}-59^{\circ} 45^{\prime} \mathrm{W}\right)$ y según nuestra revisión no ha sido coleccionada en otras localidades de los Sistemas de Tandilia o Ventania. Es muy abundante en sectores húmedos en un cerro, y comparte el área con un pajonal de Paspalum quadrifarium Lam. en el pie del otro cerro en un área de descarga de agua (Valicenti et al., 2012). También se ubica en pequeños manchones en ambientes disturbados y húmedos.

Habita en México, Colombia, Bolivia, Paraguay, Brasil, Uruguay y nordeste de la Argentina: Buenos Aires, Chaco, Corrientes, Entre Ríos, Formosa, Misiones, Santa Fe (Welker et al., 2015; Flora Argentina, 2019). Para la provincia de Buenos Aires era considerada rara en el Delta (Cabrera, 1970; Cabrera et Zardini, 1978), donde en algunas islas forma maciegas o pajales robustos (Burkart, 1957). Los estudios de la vegetación de la Depresión del Salado (Vervoorst, 1967) permitieron ampliar el conocimiento de su distribución en la provincia. En ese trabajo se la mencionó para las proximidades de Espigas, norte del partido de Olavarría (36 $26^{\circ}$ 'S $-60^{\circ}$ 39'W), en un pajonal mixto de Paspalum quadrifarium Lam. y Spartina sp., también en flechillares y terraplenes de la depresión del Salado, a fines de la década de 1950. Fue coleccionada en el partido de Las Flores (Ea. El Toro, Rosas, 36 ${ }^{\circ} 09^{\prime} \mathrm{S}, 5^{\circ} 5^{\circ}$ 'W) en 1966 (Herbario BA, Portal de Datos Biológicos, 2019). En la revisión del género Erianthus (Molina, 1981) se menciona su distribución en Buenos Aires sin consignar regiones dentro de la provincia, ni tampoco el límite sur preciso del rango de distribución geográfica.

La población hallada en las sierras de Azul sería la más austral conocida y por lo tanto se puede considerar al Sistema de Tandilia el límite sur de su distribución en América. 
C. O. D'Alfonso et al., Novedades sobre distribución de especies en Tandilia
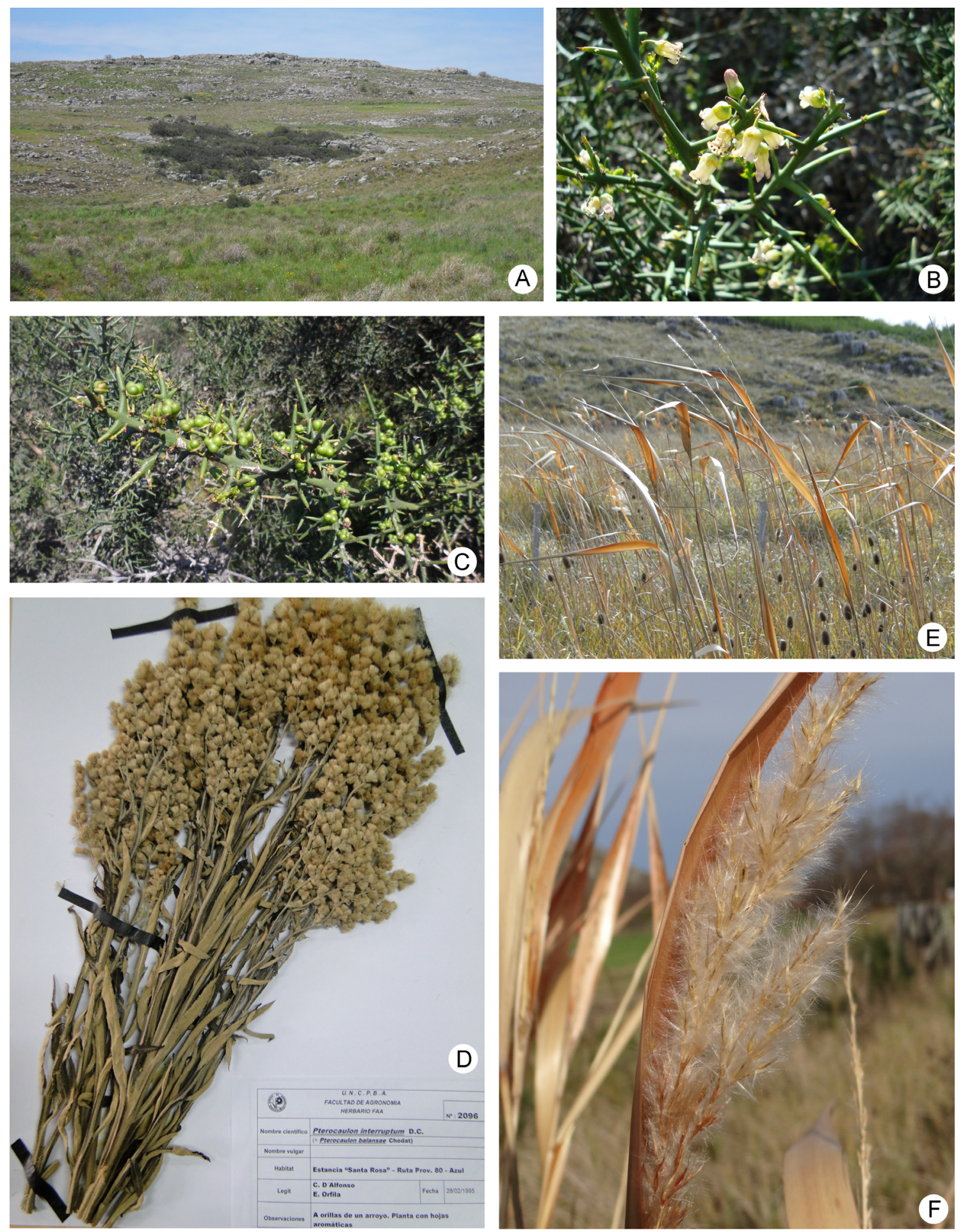

Fig. 1. Colletia spinosissima. A: Ambiente serrano. B: Rama con flores. C: Rama con frutos. Pterocaulon balansae. D: Planta (Orfila \& D'Alfonso s.n.; FAA 2036). Saccharum trinii. E: Población serrana. F: Panoja de espiguillas.

Fig. 1. Colletia spinosissima. A: Serrano ambient. B: Branch with flowers. C: Branch with fruits. Pterocaulon balansae. D: Plant (Orfila \& D'Alfonso w.n.; FAA 2036). Saccharum trinii. E: Serrana population. F: Panicle of spikelets. 
Material examinado: ARGENTINA. Buenos Aires: Partido de Azul, Sierras de Azul, Boca de las Sierras, $37^{\circ} 03^{\prime}$ 44,09'S, 59 45' 25,10'W, 08-IX-1994, D'Alfonso s.n. (FAA 3642); ib., Ea. Los Cerrillos, $37^{\circ}$ 03' 37,83'S, 59 44' 53,61'W, 15-VII-1999, D'Alfonso \& Farina s.n. (FAA 2863); ib., Ruta P. 80, cerca cerro La Armonía, 37 $03^{\circ}$ '44,09'S, $59^{\circ} 45^{\prime}$ 25,10'W, 27-IV-2006, Farina, s.n. (FAA 4602); ib., Ruta P. 80, en borde de ruta, $37^{\circ} 03$ ' 39,3"S 59 45' 25,3”W, 6-XII-2018, Scaramuzzino et al. s.n. (FAA 8846); ib., 16-VII-2019, D’Alfonso \& Piazza s.n. (FAA 10150).

\section{Discusión}

El desconocimiento de la presencia de estas especies en el Sistema serrano de Tandilia puede deberse a que existen escasas colecciones botánicas de esta región. El centro y sur bonaerense han sido consideradas áreas pobremente muestreadas, sin grandes colecciones biológicas en el pasado (Guerrero et Agnolin, 2016).

La presencia de $C$. spinosissima en distintos sectores del Sistema de Tandilia iría en el sentido de la hipótesis de Frenguelli (1941) que suponía que en otro tiempo la zona serrana estuvo más poblada de especies leñosas y vinculada con una más extendida "faja de monte" (el distrito del tala de la provincia fitogeográfica del Espinal según Cabrera, 1976), conclusiones a las que había llegado teniendo en cuenta la distribución de otra especie del género Colletia [C. paradoxa (Spreng.) Escal.], que se habría extendido hasta Olavarría, en el noroeste del sistema. Tal vez no fuera esa la especie que llegaba hasta Olavarría, sino $C$. spinosissima o bien convivían. Existen referencias a la presencia de esta especie en Tandilia en el siglo XIX (Holmberg, 1884), por lo que no se trataría de una expansión reciente en su distribución debida al cambio climático. Por el contrario, es probable que en el Sistema de Tandilia haya sido más abundante y que su reducción en el presente esté relacionada no sólo con factores climáticos del siglo XIX o anteriores, sino también antrópicos, como el manejo ganadero. Según comunicación de productores de la zona, en un establecimiento agropecuario del partido de Azul se intentó eliminar estos arbustales mediante fuego pero esto no fue posible. También puede haber sido utilizada como combustible, como lo ha sido en otros sitios de su distribución (Tortosa, 1989). Asimismo la actividad minera puede haber contribuido a su retracción.

La distribución de esta especie en la provincia de Buenos Aires parecería haber disminuido su hábitat. Las causas pueden haber sido los cambios en las costas bonaerenses durante el Holoceno (Violante et al., 2001) o anteriores, o la retracción de los talares por causas climáticas o antrópicas durante los últimos siglos. Asimismo, es posible que la disyunción entre el norte y el sur de Buenos Aires sea sólo aparente por ausencias metodológicas, ya sea por falta de relevamientos detallados en el espacio o en el tiempo. La dispersión por semillas en trayectos tan grandes parece improbable, si bien los frutos se abren activamente a la madurez y las semillas son expelidas a distancia (Tortosa, 1989), o tal vez intervengan otros agentes de dispersión como animales mediante corredores biológicos.

El análisis de la distribución bonaerense de esta especie durante el siglo XX en la literatura botánica es confuso. En la Flora de la provincia de Buenos Aires (Cabrera, 1965) se indicó su presencia en el "sudoeste" de la provincia, puede ser un error de imprenta y debió decir "sudeste", pero en trabajos posteriores no se consignó ninguna de las dos localizaciones (Tortosa, 1989, 1995). Asimismo, Parodi cuando describió la composición y distribución de los talares consignó en una publicación que C. spinosissima se extendía hasta General Lavalle (Parodi, 1940a), mientras que en otra, tal vez con otros datos, señaló que formaba "enormes matorrales que seguían con el tala hasta cerca de Mar Chiquita" (Parodi, 1940b). Tampoco esta distribución fue confirmada posteriormente (Tortosa, 1989). Los registros aparentemente recientes en el extremo sudeste del sistema (Laguna de los Padres, Sierra La Barrosa, Reserva Paitití) como también en el partido de la Costa, podría deberse a un avance hacia el sur desde la ribera del Plata y posteriormente hacia el sudeste el sistema de 
Tandilia, tal vez recuperando una antigua área de ocupación.

Pterocaulon balansae presenta en la provincia de Buenos Aires una distribución parecida a la de C. spinosissima (si bien en ambientes diferentes), tanto en el noreste (Cabrera et Ragonese, 1978) como en el Sistema de Tandilia, aunque las poblaciones de Pterocaulon conocidas en las sierras son muy distantes entre sí. A diferencia de $C$. spinosissima, el papus podría haber facilitado la dispersión a grandes distancias pero no se descarta la existencia de otras poblaciones en sitios poco accesibles a las investigaciones botánicas. Sería importante contar con un mayor número de colectas o análisis filogeográficos, para poder concluir si las poblaciones entre el norte y el sur de la provincia provienen de la fragmentación de una población antecesora, o si es un avance de las últimas décadas debido al cambio climático con la contribución de la anemocoria como método de dispersión.

En cuanto a $S$. trinii, la distribución es diferente en la provincia de Buenos Aires con respecto a las otras especies mencionadas. Es llamativo que durante la mayor parte del siglo XX sólo fuera conocida hasta el Delta del Paraná (Cabrera, 1970). Podría deberse a que sus poblaciones fueran escasas en otras regiones de la provincia e inaccesibles a la mayoría de los coleccionistas botánicos. Tal vez las poblaciones actuales sean un relicto de la vegetación de períodos de mayores temperaturas como por ejemplo el Máximo Térmico del Holoceno (7500-4500 años AP), cuando elementos faunísticos subtropicales llegaron hasta Azul (Tonni, 2017). Pero también una alternativa posible es que estas poblaciones hayan avanzado hacia el sur debido a los incrementos en la precipitación y temperatura que ocurrieron durante el siglo pasado (Guerrero et Agnolin, 2016; Tonni, 2017). Los pelos largos en las espiguillas probablemente contribuyan a su dispersión por el viento. Asimismo es probable que la distribución haya sido afectada por el factor antrópico. Sus poblaciones pueden haber disminuido al fragmentarse los pajonales de Paspalum quadrifarium (Gandini et al., 2019) con los que se encontraba asociada (Vervoorst,
1967), o por el contrario, su avance puede haberse facilitado por las rutas, caminos y vías de la red de ferrocarriles que actuarían como corredores biológicos, dado que se la ha encontrado en terraplenes (Vervoorst, 1967) y bordes de rutas.

\section{Conclusiones}

El Sistema de Tandilia es el hábitat de las poblaciones más australes de C. spinosissima, $P$. balansae y $S$. trinii. Este trabajo aporta nuevos datos para estudiar el rol de este sistema serrano en la biogeografía de la región pampeana actual e histórica. A su vez, para cada una de las especies analizadas, a partir del conocimiento de su límite sur de distribución, podrán inferirse nuevos datos de sus requerimientos ambientales.

El conocimiento de las distribuciones marginales plantea un nuevo objetivo de conservación de la biodiversidad. Aunque tradicionalmente se consideraba de poco valor para la conservación a las poblaciones en la periferia del rango de extensión geográfica de cada especie vegetal o animal (Channell, 2004), en los últimos años se ha puesto en valor la conservación de las poblaciones periféricas (Channell et Lomolino, 2000). Entre otros aspectos, se considera que están "adaptadas" a futuros cambios en el ambiente (Channell, 2004), por formar parte potencial de todo el acervo genético de las mismas. Por lo tanto, sería importante establecer estrategias para la conservación de estas poblaciones, como por ejemplo la creación de reservas o parques nacionales que las contengan.

\section{Agradecimientos}

Este trabajo fue realizado con financiamiento de la Universidad Nacional del Centro de la provincia de Buenos Aires, mediante los Proyectos 03/A219 y 03/A216. Todos los autores son integrantes del núcleo de investigación NUCEVA. Se agradece a los revisores las sugerencias que contribuyeron a mejorar el manuscrito. 


\section{Bibliografía}

ALONSO, S. I., I. R. GUMA, M. C. NUCIARI \& A. VAN OLPHEN. (2009). Flora de un área de la Sierra La Barrosa (Balcarce) y fenología de especies con potencial ornamental. Revista de la Facultad de Ciencias Agrarias UNCuyo 41: 23-44.

APODACA, M. J. \& E. L. GUERRERO. (2019). ¿Por qué se expande hacia el sur la distribución geográfica de Tillandsia recurvata (Bromeliaceae)? Boletín de la Sociedad Argentina de Botánica 54: 255-261. http://dx.doi. org/10.31055/1851.2372.v54. n2.24371

BAYÓN, N. \& J. HURRELL. (2013). Pterocaulon. En HURRELL, J. (ed.), Asteraceae. Flora Rioplatense, parte 2, vol. 7a, pp. 186-196. Sociedad Argentina de Botánica, Corrientes.

BURKART, A. (1957). Ojeada sinóptica sobre: La vegetación del Delta del Río Paraná. Darwiniana 11: 457-561.

CABRERA, A. L. (1941). Compuestas bonaerenses. Revisión de las compuestas de la Provincia de Buenos Aires, la Capital Federal y la Isla Martín García. Revista Museo de La Plata, Botánica 4: $1-450$.

CABRERA, A. L. (1965). Flora de la Provincia de Buenos Aires. Colección Científica INTA tomo 4, parte 4. Instituto Nacional de Tecnología Agropecuaria, Buenos Aires.

CABRERA, A. L. (1968). Flora de la Provincia de Buenos Aires. Colección Científica INTA tomo 4, parte 1. Instituto Nacional de Tecnología Agropecuaria, Buenos Aires.

CABRERA, A. L. (1970). Flora de la Provincia de Buenos Aires. Colección Científica INTA tomo 4, parte 2. Instituto Nacional de Tecnología Agropecuaria, Buenos Aires.

CABRERA, A. (1976). Regiones fitogeográficas argentinas. Enciclopedia argentina de agricultura y jardinería. Tomo 2. 2a edición. Fascículo 1. ACME S. A., Buenos Aires.

CABRERA, A. L. \& G. DAWSON. (1944). La selva marginal de Punta Lara en la ribera argentina del Río de La Plata. Revista del Museo de La Plata, Botánica 22: 1-382.

CABRERA, A. L. \& A. M. RAGONESE. (1978). Revisión del género Pterocaulon (Compositae). Darwiniana 21: 185-257.

CABRERA, A. L. \& E. ZARDINI. (1978). Manual de la flora de los alrededores de Buenos Aires. 755 pp. ACME S. A., Buenos Aires.
CABRERA, A., J. CRISCI, G. DELUCCHI, S. FREIRE, D. GIULIANO, L. IHARLEGUI, L. KATINAS, A. SÁENZ, G. SANCHO \& E. URTUBEY. (2000). Catálogo ilustrado de las Compuestas (= Asteraceae) de la Provincia de Buenos Aires, Argentina: Sistemática, Ecología y Usos. COBIOBO $\mathrm{N}^{\circ} 2$, PROBIOTA 1: 1-137. U.N.L.P., La Plata.

CHANNELL, R. (2004). The conservation value of peripheral populations: the supporting science. Proceedings of the Species at Risk 2004 Pathways to Recovery Conference. 1-17. Victoria, Canadá: Organizing Committee.

CHANNELL, R. \& M. V. LOMOLINO. (2000). Dynamic biogeography and conservation of endangered species. Nature 403: 84-86. https://doi. org $/ 10.1038 / 47487$

CINGOLANI, C. A. (2011). The Tandilia System of Argentina as a southern extension of the Río de la Plata craton: an overview. International Journal of Earth Sciences 100: 221-242. https://doi.org/10.1007/s00531-010-0611-5

DOERING, A. \& P. LORENTZ. (1916). Recuerdos de la Expedición al Río Negro. Boletín de la Academia Nacional de Ciencias de Córdoba 21: 301-386.

ECHEVERRÍA, M., S. ALONSO \& V. COMPARATORE. (2017). Survey of the vascular plants of Sierra Chica, the untouched area of the Paititi Natural Reserve (southeastern Tandilia mountain range, Buenos Aires province). Check List 13: 1003-1036. https://doi.org/10.15560/13.6.1003

FLORA ARGENTINA. (2019). Disponible: http://www. floraargentina.edu.ar (Consulta 14-VI-2019).

FRANGI, J. (1975). Sinopsis de las comunidades vegetales y el medio de las Sierras de Tandil (Provincia de Buenos Aires). Boletín de la Sociedad Argentina de Botánica 16: 293-319.

FREIRE, S. E. \& L. IHARLEGUI. (2015). Pterocaulon Elliot. En ZULOAGA, F. O., M. J. BELGRANO \& A. M. ANTON (eds.), Flora Argentina, Asteraceae 7(2): 272-282.

FRENGUELLI, J. (1941). Rasgos principales de Fitogeografia Argentina. Revista Museo La Plata, Nueva serie, Botánica 3: 65-181.

GUERRERO, E. L. \& F. L. AGNOLIN. (2016). Recent changes in plant and animal distribution in the southern extreme of the Paranaense biogeographical province (northeastern Buenos Aires province, Argentina): Ecological responses to climate change? Revista del Museo Argentino de Ciencias Naturales nueva serie 18: 9-30. https://doi.org/10.22179/REVMACN.18.428 
GUERRERO, E., J. DESCHAMPS \& E. TONNI. (2018). La Selva Marginal de Punta Lara, ¿relicto o colonización reciente? Revista del Museo de La Plata 3: 348-367. https://doi.org/10.24215/25456377e064

HOLMBERG, E. L. (1884). Viajes a las Sierras del Tandil y de la Tinta. Actas de la Academia Nacional de Ciencias en Córdoba 5: 1-58.

KATINAS, L. (1994). Un nuevo género de Nassauviinae (Asteraceae, Mutisieae) y sus relaciones cladísticas con los géneros afines de la subtribu. Boletín de la Sociedad Argentina de Botánica 30: 59-70.

KATINAS, L. \& J. V. CRISCI. (2008). Reconstructing the biogeographical history of two plant genera with different dispersion capabilities. Journal of Biogeography 35: 1374-1384. https://doi.org/10.1111/j.1365-2699.2007.01874.x.

GANDINI, M. L., B. D. LARA, L. B. MORENO, M. A. CAÑIBANO \& P. A. GANDINI. (2019). Trends in fragmentation and connectivity of Paspalum quadrifarium grasslands in the Buenos Aires province, Argentina. PeerJ 7: e6450. http://doi.org/10.7717/peerj.6450

IPCC. (2018). Global warming of $1.5^{\circ} \mathrm{C}$. An IPCC Special Report on the impacts of global warming of $1.5^{\circ} \mathrm{C}$ above pre-industrial levels and related global greenhouse gas emission pathways, in the context of strengthening the global response to the threat of climate change, sustainable development, and efforts to eradicate poverty. https://www.ipcc. ch/sr15/ (Consulta 13-VI-2019).

MACARTHUR, R. \& E. WILSON. (1969). The theory of island biogeography. 1a ed. Princeton University Press, New Jersey (US).

MOLINA, A. M. (1981). El género Erianthus (Gramineae) en la Argentina y países limítrofes. Darwiniana 23: 559-585.

OLLERTON, J., G. CHANCELLOR \& J. VAN WYHE. (2012). John Tweedie and Charles Darwin in Buenos Aires. Notes and Records of The Royal Society 66: 115-124.

https://doi.org/10.1098/rsnr.2011.0052.

PARODI, L. R. 1940a. Distribución geográfica de los talares de la Provincia de Buenos Aires. Darwiniana 4: 33-56.

PARODI, L. R. (1940b). Los bosques naturales de la provincia de Buenos Aires. Anales Academia Nacional Ciencias Exactas Físicas y Naturales 7: 79-90.

POPP, M., A. GIZAW, S. NEMOMISSA, J. SUDA \& C. BROCHMANN. (2008). Colonization and diversification in the African 'sky islands' by Eurasian Lychnis L. (Caryophyllaceae). Journal of Biogeography 35: 1016-1029.

https://doi.org/10.1111/nph.13937.

PORTAL DE DATOS DE BIODIVERSIDAD ARGENTINA. (2019). Disponible: https://datos. sndb.mincyt.gob.ar/ (Consulta 14-VI-2019).

ROBSON, N. K. B. (1990). Studies in the genus Hypericum L. (Guttiferae). 8. Sections 29. Brathys (part 2) and 30. Trigynobrathys. Bulletin of the British Museum (Natural History), Botany 20: 1-151.

SPEZIALE, K. \& C. EZCURRA. (2014). Rock outcrops as potential biodiversity refugia under climate change in North Patagonia. Plant Ecology \& Diversity 8: 353-361.

https://doi.org/10.1080/17550874.2014.983200

TERUGGI, M. E. \& J. O. KILMURRAY. (1980). Sierras Septentrionales de la Provincia de Buenos Aires. En: Geología Regional Argentina. Academia Nacional de Ciencias, Córdoba 2: 919956.

THIERS, B. (2019). [permanentemente actualizado] Index Herbariorum: a global directory of public herbaria and associated staff. New York Botanical Garden's Virtual Herbarium, http://sweetgum. nybg.org/ih. (Consulta 20/06/2019).

TONNI, E. P. (2017). Cambios climáticos en la región pampeana oriental durante los últimos 1000 años. Una síntesis con énfasis en la información zoogeográfica. Revista del Museo de La Plata 2: 1-11. https://doi.org/10.24215/25456377e038

TORTOSA, R. D. (1989). El género Colletia (Rhamnaceae). Parodiana 5: 279-332.

TORTOSA, R. D. (1995). Rhamnaceae. En HUNZIKER, A. T. (ed.), Flora Fanerogámica Argentina. 9: 1-18.

TROPICOS. (2019). Tropicos.org. Missouri Botanical Garden. Disponible: http://www.tropicos.org. (Consulta 14-VI-2019).

VALICENTI, R., E. FARINA, C. D'ALFONSO \& R. SCARAMUZZINO. (2012). Pajonales serranos de Paspalum quadrifarium. Composición florística, origen de las especies, formas de vida y relaciones ambientales. I Jornadas Nacionales de Ambiente FCH - UNICEN, pp. 58-71. Universidad Nacional del Centro de la Provincia de Buenos Aires, Tandil.

VERVOORST, F. B. (1967). La vegetación de la República Argentina. VII. Las comunidades vegetales de la depresión del Salado. Serie Fitogeográfica $\mathrm{N}^{\mathbf{0}} 7$ INTA. 
VILLAMIL, C. \& G. MARTÍNEZ. (2014). Plantas Silvestres del Partido de Tres Arroyos. Gráfica \& Editora Independencia, Tandil. $161 \mathrm{pp}$.

VIOLANTE, R.A., G. PARKER \& J. L.CAVALLOTTO. (2001). Evolución de las llanuras costeras del este bonaerense entre la bahía Samborombon y la laguna Mar Chiquita durante el Holoceno. Revista de la Asociación Geológica Argentina 56: 51-66.

WELKER, C. A., T. T. SOUZA-CHIES, H. M. LONGHI-WAGNER, M. C. PEICHOTO, M. R. MCKAIN \& E. A. KELLOGG. (2015). Phylogenetic analysis of Saccharum s.l. (Poaceae; Andropogoneae), with emphasis on the circumscription of the South American species.
American Journal of Botany 102: 248-263. https://doi.org/10.3732/ajb.1400397

ZÁRATE, M. \& J. RABASSA. (2005). Geomorfología de la provincia de Buenos Aires. En DE BARRIO, R., R. O. ETCHEVERRY, M. F. CABALLÉ \& E. LLAMBÍAS (eds.), Geología y Recursos Naturales de la Provincia de Buenos Aires. Relatorio XVI Congreso Geológico Argentino pp. 119-138.

ZÁRATE, M. (2010). El paisaje pampeano a través del tiempo. En BERÓN, M. L. LUNA, M. BONOMO, C. MONTALVO, C. ARANDA \& M. CARRERA AIZPITARTE (eds.), Mamül Mapu: pasado y presente desde la arqueología pampeana, pp. 215-226. Editorial Libros del Espinillo, Ayacucho, Buenos Aires. 